\title{
Regio- and Stereoselective Ring-Opening of Nonactivated Amino Aziridines by Thiols and Unusual Nucleophilic Substitution of a Dibenzylamino Group
}

\author{
José M. Concellón,* ${ }^{[a]}$ Pablo L. Bernad, ${ }^{[a]}$ José Ramón Suárez, ${ }^{[a]}$ Santiago García- \\ Granda $^{[b]}$ and M. Rosario Díaz ${ }^{[b]}$ \\ ${ }^{[a]}$ Departamento de Química Orgánica e Inorgánica, \\ Facultad de Química, Universidad de Oviedo, Julián \\ Clavería, 8, 33071 Oviedo, Spain. \\ ${ }^{[b]}$ Departamento de Química Física y Analítica, Facultad de \\ Química, Universidad de Oviedo, Julián Clavería, 8, 33071 \\ Oviedo, Spain.
}

Email:jmcg@uniovi.es

\section{${ }^{13} \mathrm{C}-\mathrm{RMN}$ of 2,3 and 4 :}

$(2 S, 3 S)-N^{3}, N^{3}$-Dibenzyl-2-(pentylthio)- $N^{l}$-(propyl)butan-1,3-diamine (2a)..................... S2

$(2 S, 3 S)-N^{3}, N^{3}$-Dibenzyl-2-(cyclohexylthio)- $N^{l}$-(propyl)butan-1,3-diamine (2b).................. 2

$(2 S, 3 S)-N^{I}$-Allyl- $N^{3}, N^{3}$-dibenzyl-2-(2-phenylethylthio)butan-1,3-diamine (2c) ................. S3

$(2 S, 3 S)-N^{1}, N^{3}, N^{3}$-Tribenzyl-4-benzyloxy-2-(cyclohexylthio)butan-1,3-diamine (2d)................... S3

$(2 S, 3 S)-N^{1}, N^{3}, N^{3}$-Tribenzyl-4-benzyloxy-2-(benzylthio)butan-1,3-diamine (2e)..................... S4

$\left(2 S^{*}, 3 S^{*}\right)-N$-Allyl-5-methyl-2,3-bis(pentylthio)hexan-1-amine (3a) ................................... S4

$\left(2 S^{*}, 3 S^{*}\right)-N$-Allyl-5-methyl-2,3-bis(2-phenylethylthio)hexan-1-amine (3b)........................... S5

$\left(2 S^{*}, 3 * S\right)$ - $N$-Benzyl-5-methyl-2,3-bis(pentylthio)hexan-1-amine (3c)............................. S5

$\left(2 S^{*}, 3 S^{*}\right)-N$-Benzyl-5-methyl-2,3-bis(octylthio)hexan-1-amine (3d) .................................... S6

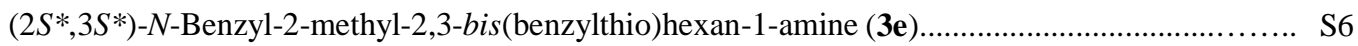

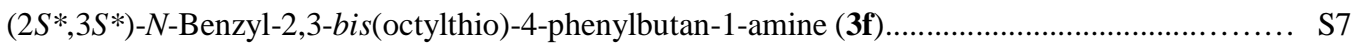

$\left(2 S^{*}, 3 S^{*}\right)-N$-Benzyl-2,3-bis(ciclohexylthio)-4-phenylbutan-1-amine (3g)............................. S7

$\left(2 S^{*}, 3 S^{*}\right)-N$-Benzyl-2,3-bis(phenylethylthio)-4-phenylbutan-1-amine (3h)........................... S8

$\left(2 S^{*}, 3 S^{*}\right)-1-N$-Benzyl- $N$-[2,3-bis(cyclohexylthio)-4-phenylbutyl)]benzamide (4g)................... S8 


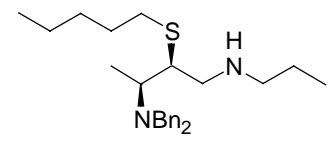

$2 a$

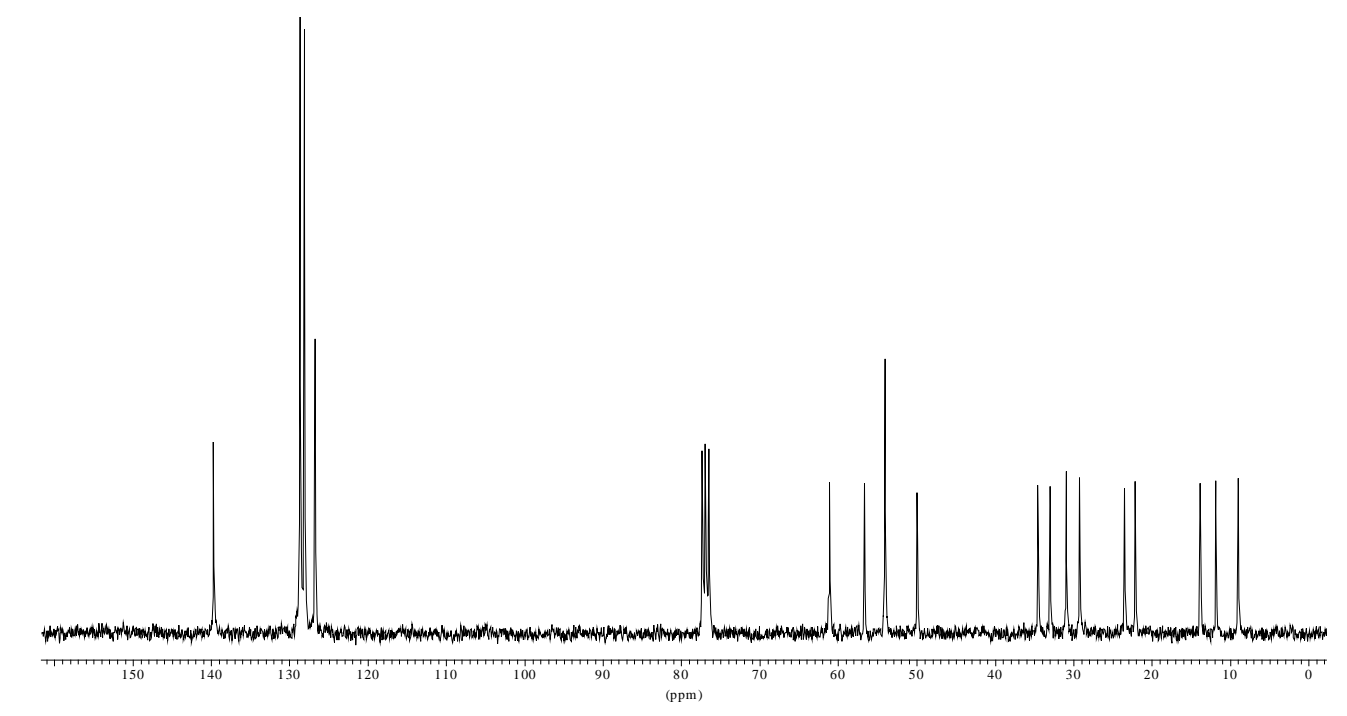

$\overbrace{\mathrm{NBn}_{2}}^{\mathrm{C}}$

$2 b$

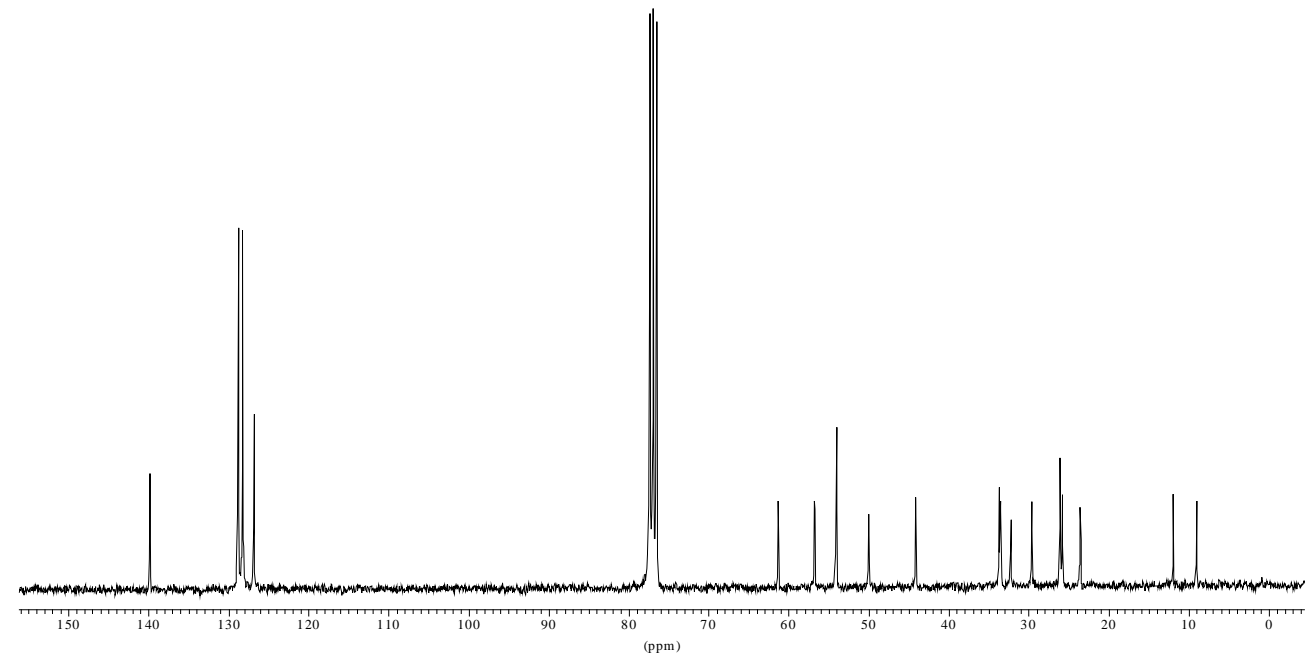


NBn $_{\mathrm{NBn}_{2}}^{\mathrm{C}}$

2c

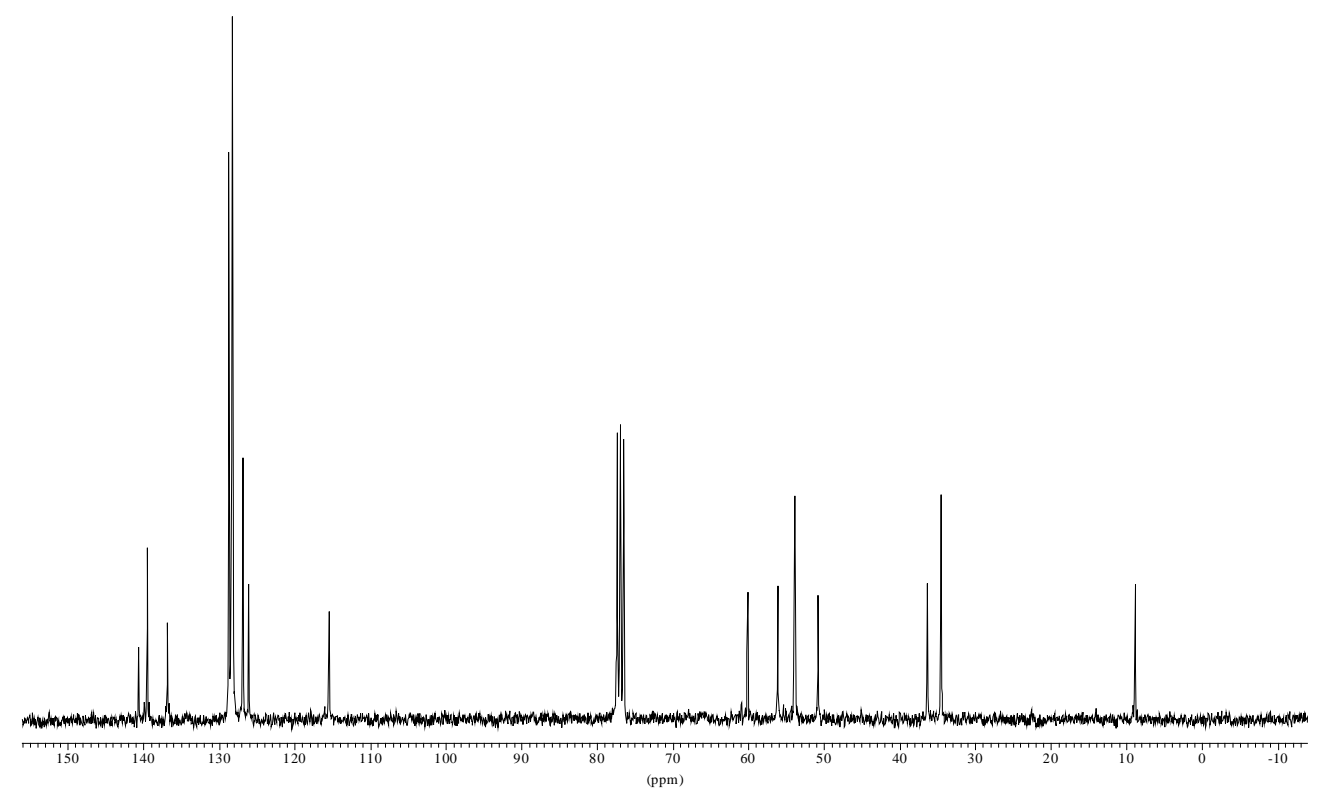
$\overbrace{\mathrm{NBn}_{2}}^{\mathrm{C}} \overbrace{\mathrm{N}}^{\mathrm{C}} \overbrace{\mathrm{Ch}}^{\mathrm{C}}$

2d

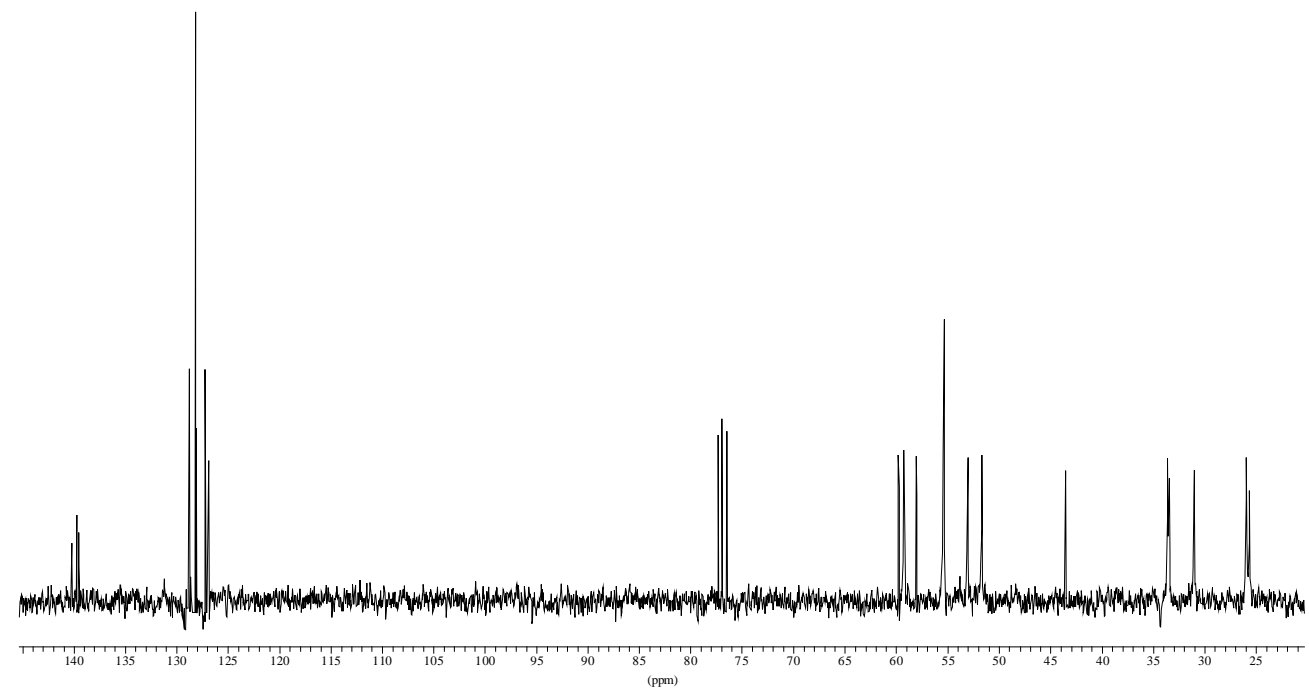




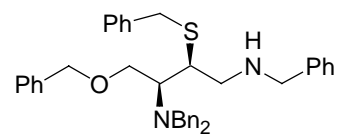

$2 e$
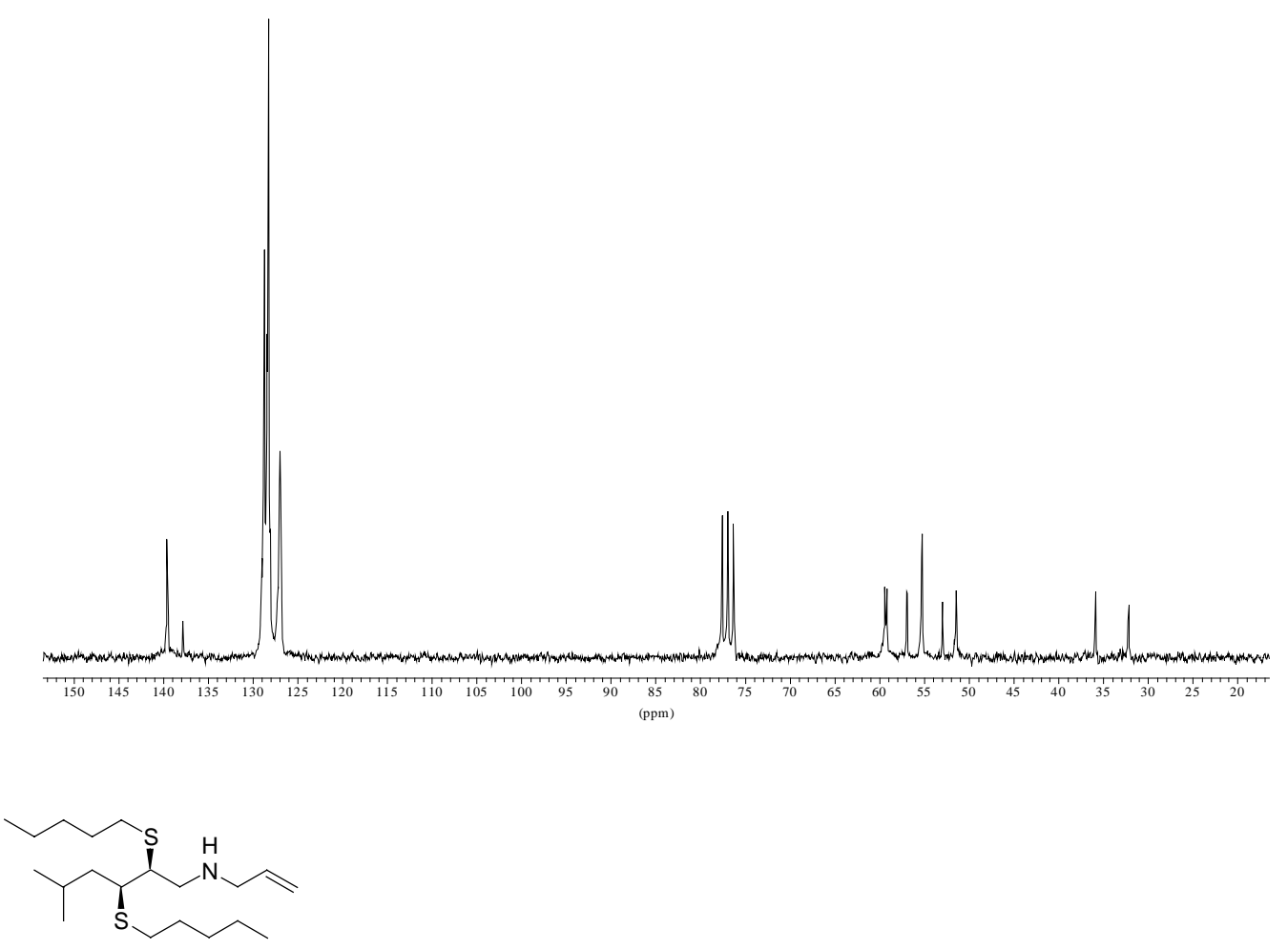

$3 a$

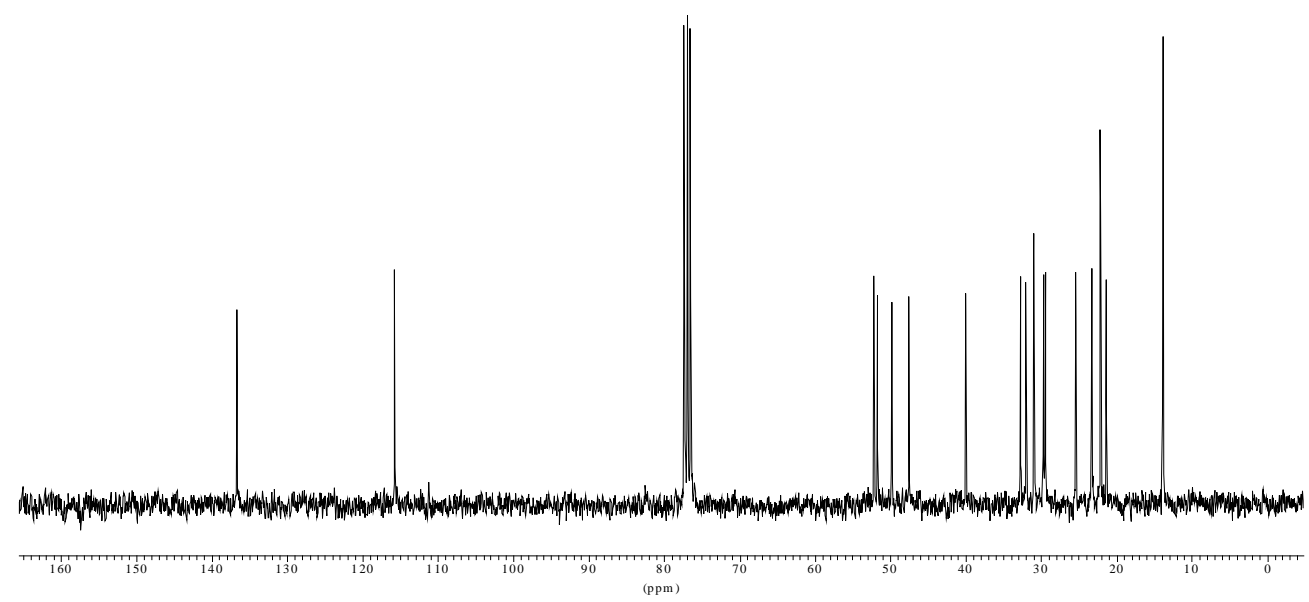




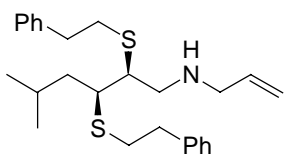

3b
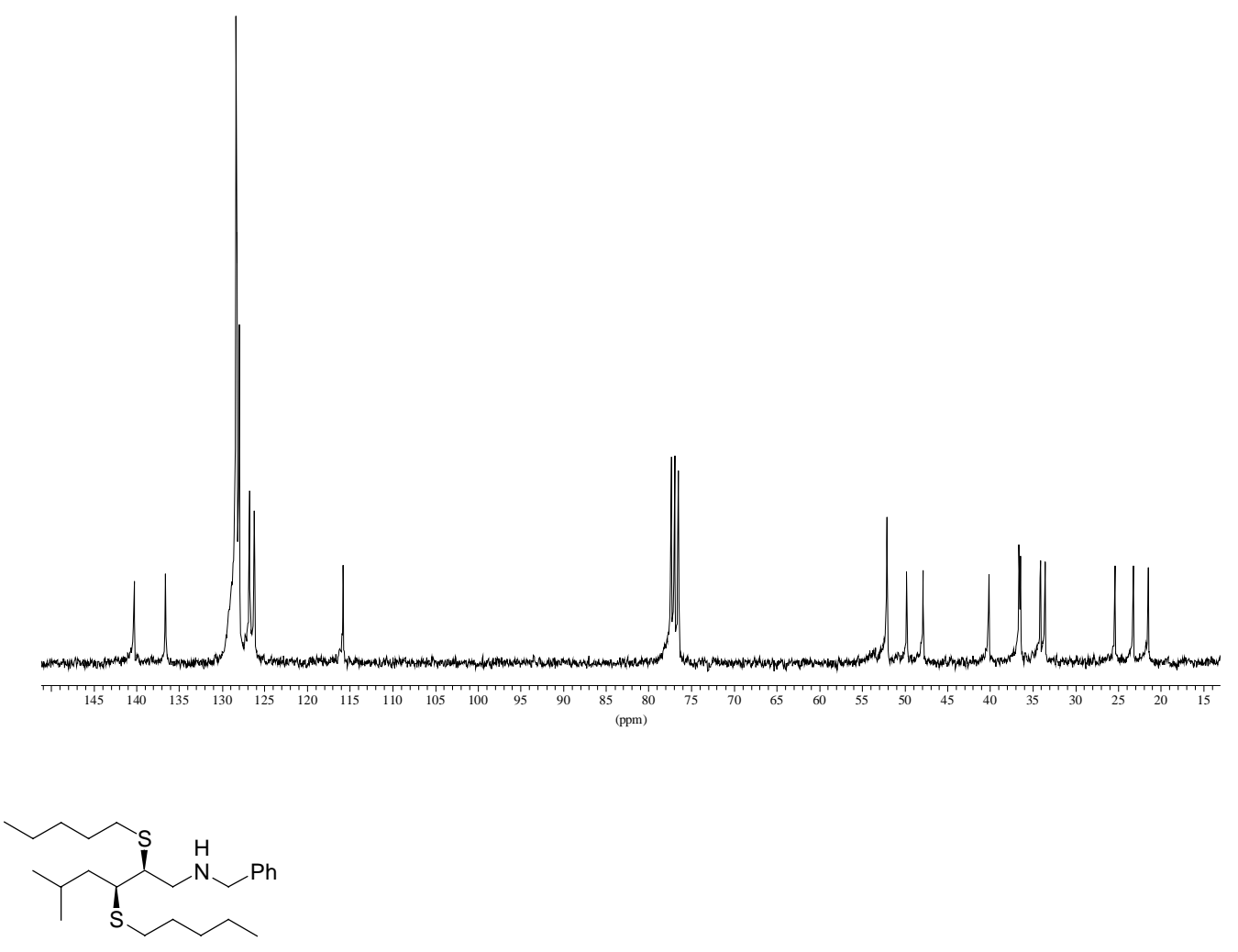

3c

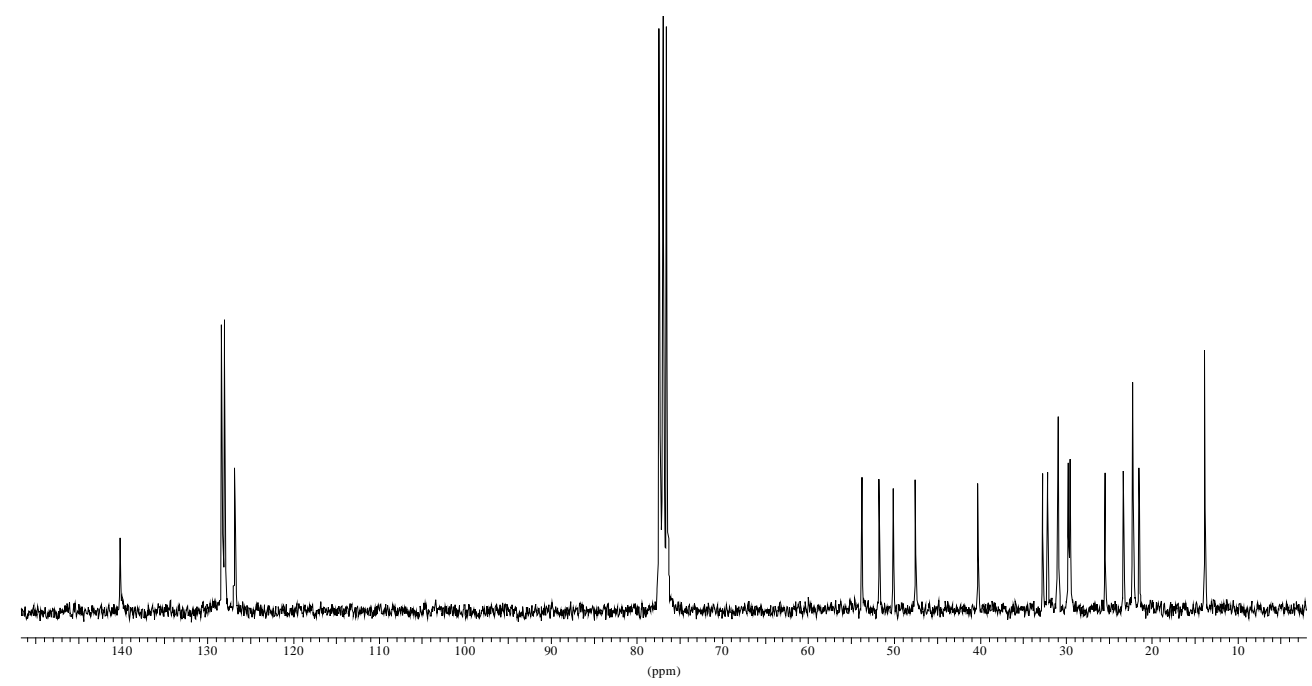




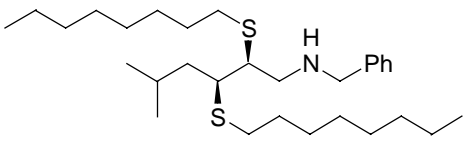

3d
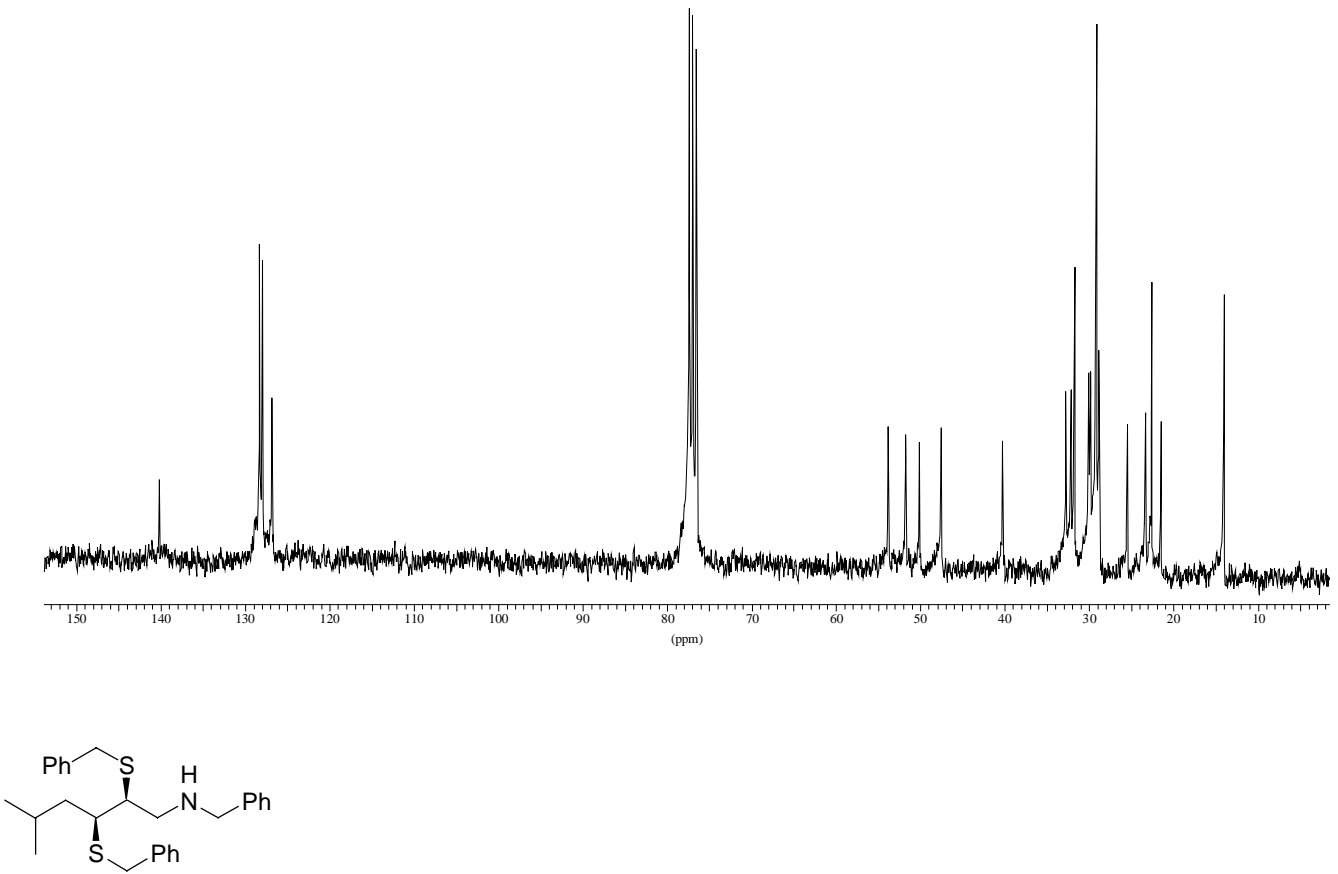

$3 e$

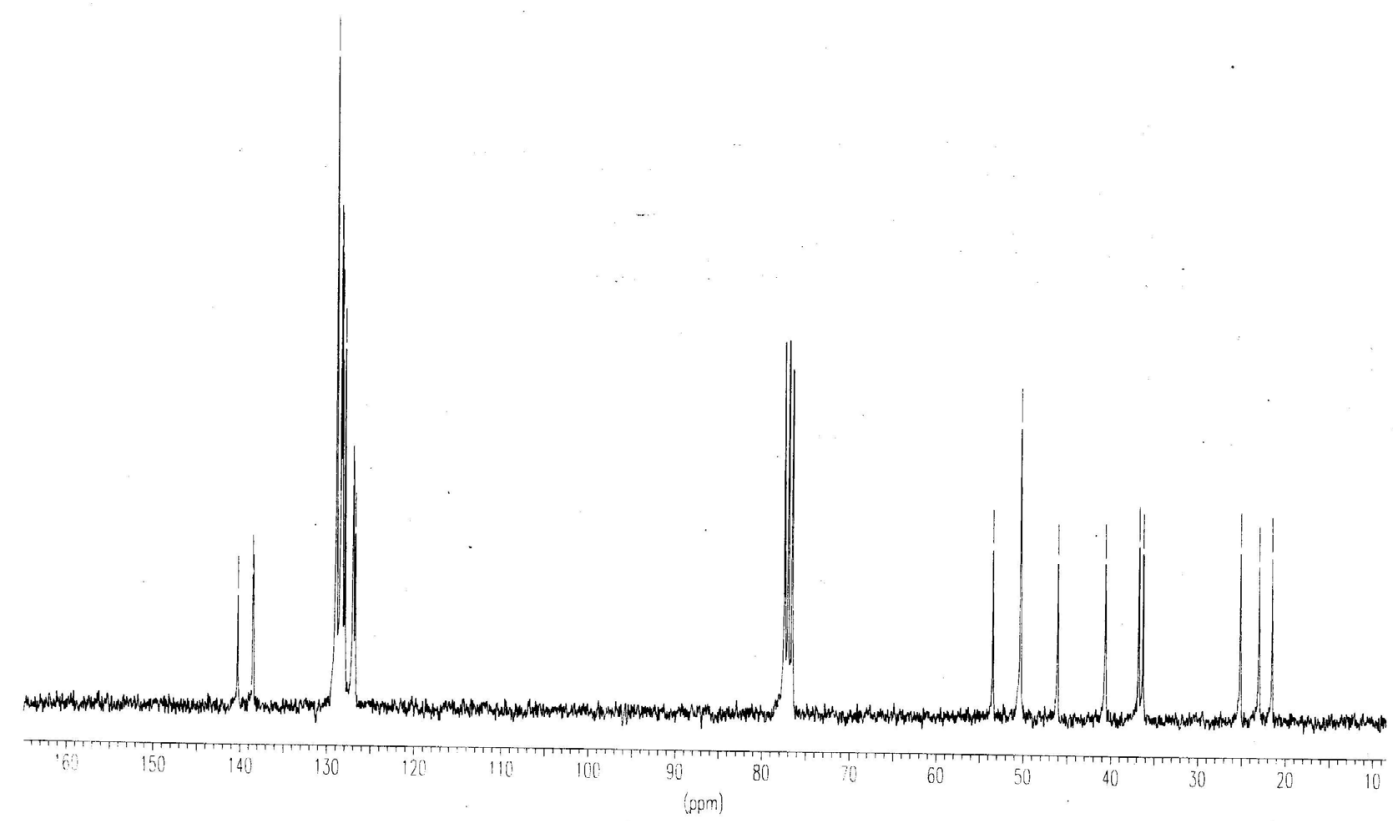




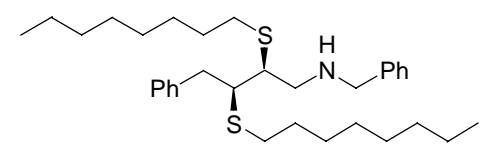

$3 f$

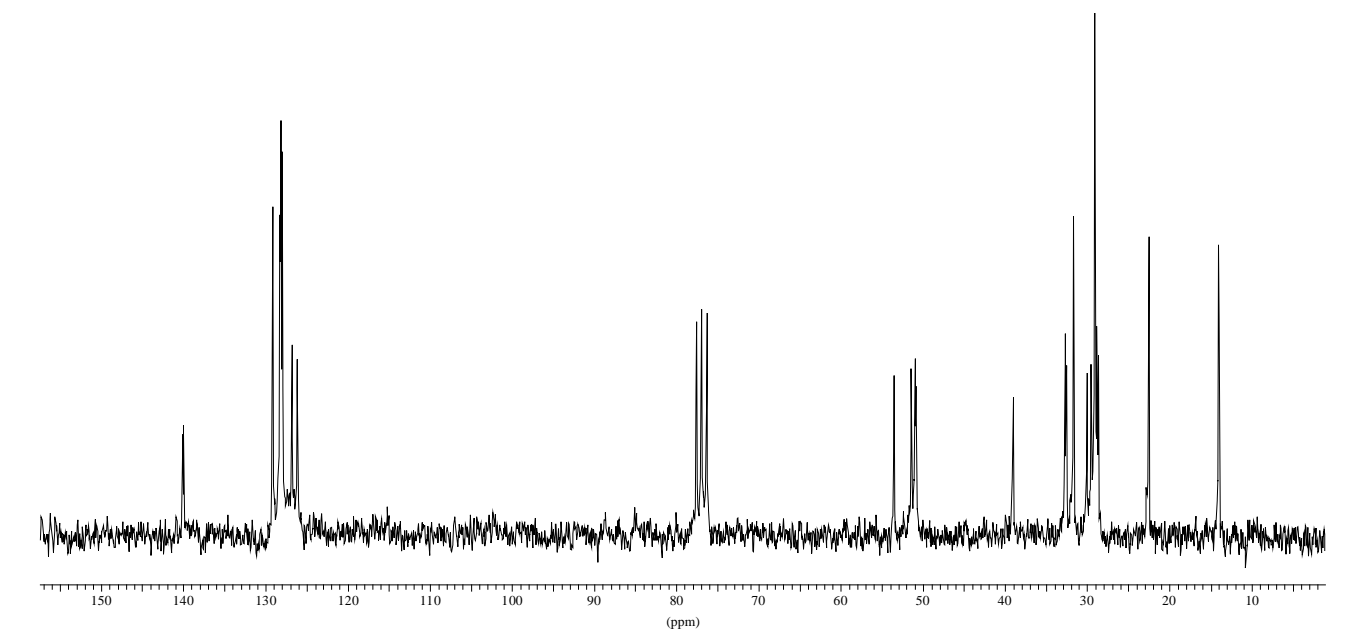<smiles>c1ccc(CNCC(S[C]2CCCCC2)C(Cc2ccccc2)SC2CCCCC2)cc1</smiles>

3g

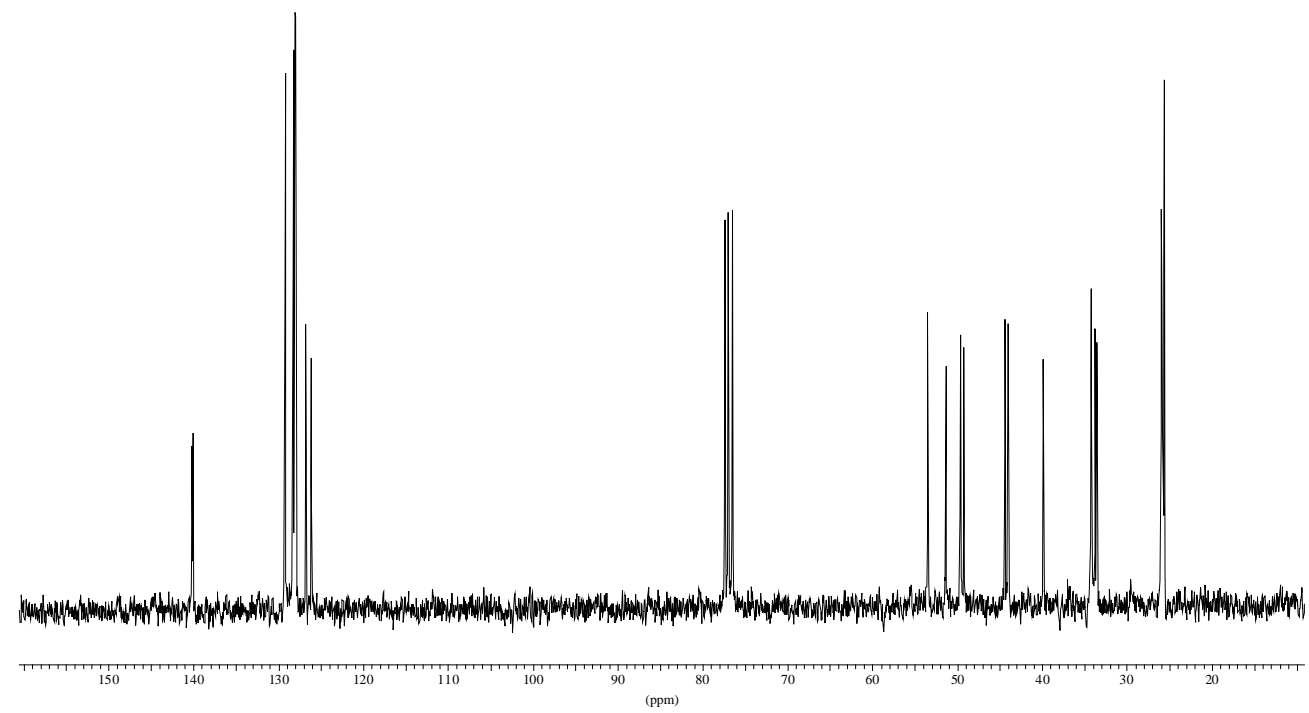




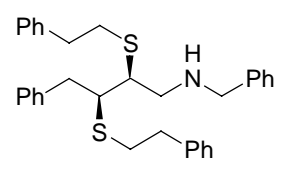

$3 h$
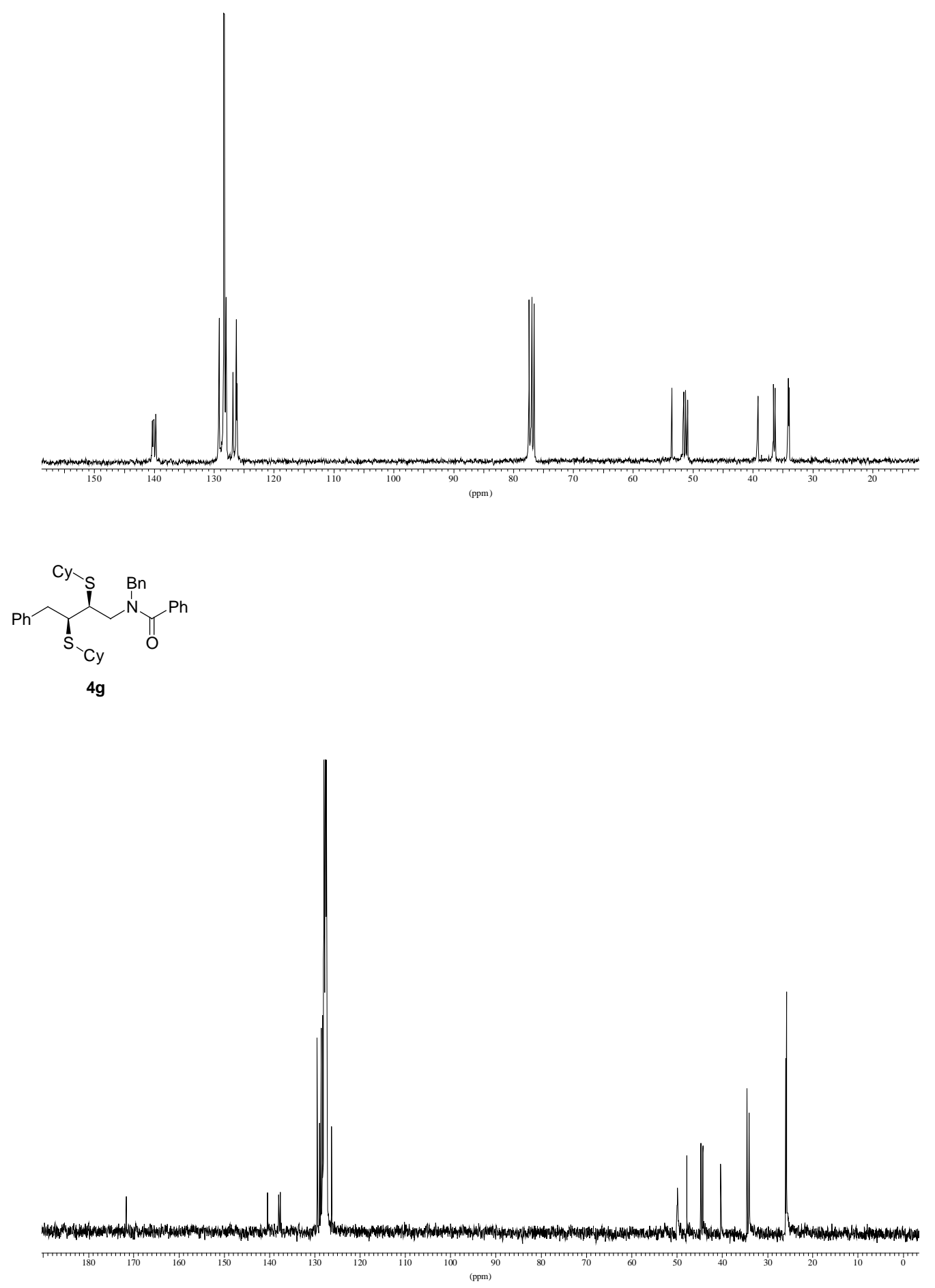